\section{Evaluation of the European Union monitoring programme on the prevalence of Listeria monocytogenes (Decision 2010/678/EU) in Turin, Italy}

\author{
Antonio Barbaro, ${ }^{1}$ \\ Annamaria Galleggiante Crisafulli, ${ }^{1}$ \\ Silvia Gallina, ${ }^{2}$ Francesca Rubinetti, ${ }^{1}$ \\ Stefano Gili, ${ }^{3}$ Sara Travaglio, ${ }^{1}$ \\ Laura Chiavacci, ${ }^{1}$ Lucia Decastelli \\ 1Struttura Complessa Epidemiologia e \\ Osservatorio Epidemiologico, Istituto \\ Zooprofilattico Sperimentale del \\ Piemonte, Liguria e Valle d'Aosta, Torino; \\ 2Struttura Complessa Controllo Alimenti \\ e Igiene delle Produzioni, Istituto \\ Zooprofilattico Sperimentale del \\ Piemonte, Liguria e Valle d'Aosta, Torino; \\ ${ }^{3}$ Dipartimento di Prevenzione, Struttura \\ Complessa Veterinaria B, Azienda \\ Sanitaria Locale TO1, Torino, Italy
}

\section{Abstract}

Listeria monocytogenes is a ubiquitous bacterium, widely distributed in the environment; morover, its ability to survive at low temperatures and form protective biofilms makes it colonise and persist in food processing plants. Epidemiological data provided by EFSA in 2009 show that ready-to-eat (RTE) food - in particular, soft and semi-soft cheese, and fishery and meat products subjected to heat treatment -represents the most likely contaminated foodstuff. For this reason, the European Commission has developed (Dec. $2010 / 678 / \mathrm{EU}$ ) a monitoring plan designed to evaluate the prevalence of $L$. monocytogenes in these products. The programme, developed in detail with reference to each member state, involved, among others, the city of Turin and has been set out from June to December 2011. The aim of this paper is to report the results obtained in the city of Turin. In total, 160 samples were analysed; only samples of smoked fish were found to be positive, while the pathogen was absent in cheese and meat products.

\section{Introduzione}

È ormai consolidato da tempo il ruolo svolto da Listeria $(L$. $)$ monocytogenes quale patogeno presente negli alimenti pronti al consumo [ready-to-eat (RTE)] conservati a temperatura di refrigerazione (Nuvolosi et al., 2006; Gandhi e Chikindas, 2007; Buchanan et al., 2004).

Ciò è dovuto alla sua ubiquitarietà, alla sua spiccata psicrofilia, alla sua capacità di creare forme di resistenza, come la formazione di biofilm, che gli permettono di resistere ai trattamenti di sanificazione; il patogeno riesce in tal modo a persistere all'interno degli stabilimenti di produzione e, attraverso le superfici di lavoro, a contaminare gli alimenti finiti, diventando spesso causa di contaminazione crociata tra alimenti diversi (Kalmokoff et al., 2001).

Per questi motivi, il Reg. CE 2073/05 (Commissione Europea, 2005) e successive modificazioni e integrazioni (ss.mm.ii.) ha inserito $L$. monocytogenes come criterio di sicurezza alimentare da verificare negli alimenti RTE che sono classificati nel regolamento stesso in: i) alimenti pronti che non costituiscono terreno favorevole alla crescita di $L$. monocytogenes [alimenti con $\mathrm{pH} \leq 4.4$ e water activity $\left(\mathrm{a}_{\mathrm{w}}\right) \leq 0,92 ;$ con $\mathrm{pH} \leq 5.0$ e $\mathrm{a}_{\mathrm{w}} \leq 0,94$ o con periodo di conservabilità inferiore a 5 giorni], e in ii) alimenti pronti che costituiscono terreno favorevole alla crescita di $L$. monocytogenes (tutti gli altri). Per gli alimenti della seconda categoria il metodo di analisi e conseguentemente il limite per classificare come adatto/non adatto al consumo sono subordinati alla capacità del produttore di dimostrare che il prodotto non supererà il limite di $100 \mathrm{ufc/g}$ durante il periodo di conservabilità.

A livello comunitario, il Community Summary Reports on Trends and Sources of Zoonoses in the EU (EFSA, 2009) riporta le percentuali di positività negli alimenti RTE prelevati in fase di commercializzazione: le matrici maggiormente contaminate risultano essere i formaggi molli e semi-molli $(1,1 \%)$, i prodotti della pesca $(1,0 \%)$ e i prodotti a base di carne sottoposti a trattamento termico $(0,3 \%)$.

Sempre secondo i dati EFSA, tra i prodotti della pesca trasformati, il salmone affumicato, confezionato sottovuoto 0 in atmosfera modificata, risulta quello maggiormente contaminato ed è considerato una potenziale fonte di infezione per l'uomo.

La contaminazione del salmone affumicato da parte di $L$. monocytogenes può avvenire in diverse fasi della lavorazione (Rørvik, 2000) e poiché una delle fonti è rappresentata dalla cute e dalla cavità intestinale del salmone stesso, durante le fasi di eviscerazione, decapitazione e filettatura, il batterio può essere trasferito sulle superfici e sulle attrezzature diventando fonte di contaminazione lungo tutte le fasi del processo produttivo (Porsby et al., 2008).

Analogamente a quanto si verifica per il salmone affumicato, anche per ciò che attiene $\mathrm{i}$ formaggi e i prodotti a base di carne, la contaminazione può derivare direttamente dalla materia prima, così come dagli ambienti di lavorazione e stagionatura.

In queste particolari categorie di prodotti
Correspondence: Antonio Barbaro, Struttura Complessa Epidemiologia e Osservatorio Epidemiologico, Istituto Zooprofilattico Sperimentale del Piemonte, Liguria e Valle d'Aosta, via Bologna 148, 10154 Torino, Italy. Tel: +39.011.2686346 - Fax: +39.011 .2485831 .

E-mail: antonio.barbaro@izsto.it

Key words: Listeria monocytogenes, Ready-to-eat food, Survey, Microbiological criteria.

Received for publication: 15 January 2013.

Revision received: 8 March 2013.

Accepted for publication: 12 March 2013.

This work is licensed under a Creative Commons Attribution 3.0 License (by-nc 3.0).

(C) Copyright A. Barbaro et al., 2013

Licensee PAGEPress, Italy

Italian Journal of Food Safety 2013; 2:e22

doi:10.4081/ijfs.2013.e22

RTE , inoltre, la moltiplicazione di L. monocytogenes può avvenire anche durante il periodo di conservazione a temperatura di refrigerazione; non sempre, infatti, le temperature dei frigoriferi domestici e di rivendite al dettaglio corrispondono a quelle raccomandate dai produttori $\left(+4^{\circ} \mathrm{C}\right)$, così come dimostrato in diversi studi (Garrido et al., 2010; Murru et al., 2011).

Per tali motivi e considerando il potenziale rischio di $L$. monocytogenes nel pesce affumicato, nei formaggi molli e semi-molli e nei prodotti a base di carne sottoposti a trattamento termico, la Commissione Europea, con la Decisione 678 del 5 novembre 2010 (Commissione Europea, 2010), ha istituito, per l'anno 2011, un piano di sorveglianza specifico teso a valutare la prevalenza di $L$. monocytogenes sul territorio comunitario nelle tre tipologie di alimenti RTE considerate maggiormente a rischio.

\section{Materiali e Metodi}

Il piano di sorveglianza comunitario specifica le caratteristiche e le finalità del monitoraggio. In particolare, sono definite le categorie di alimenti da sottoporre a campionamento (pesce affumicato a caldo 0 a freddo 0 pesce di tipo gravad lax, imballato, non congelato; formaggi a pasta molle o semi-molle, ad eccezione dei formaggi freschi; prodotti a base di carne soggetti a trattamento termico, imballati), le modalità di campionamento e le analisi da effettuare.

Inoltre, è dato mandato a ciascuno Stato Membro di predisporre uno specifico piano nazionale di campionamento (stratificato proporzionale). 
Alla città di Torino sono stati attribuiti 160 campioni di alimenti RTE da prelevare presso esercizi di vendita al dettaglio (minimarket, discount, supermercati e ipermercati) selezionati casualmente e inseriti in un sistema informativo ad hoc. I campioni sono stati prelevati dal Gruppo Progetto Sicurezza Alimentare dell'Azienda Sanitaria Locale T01. Il piano si è svolto da giugno a dicembre 2011.

Nell'ambito di tale programma, i compiti dell'Istituto Zooprofilattico Sperimentale erano quelli di i) accertare l'idoneità dei campioni conferiti ai fini dell'analisi, anche attraverso la verifica della temperatura di trasporto; ii) eseguire le analisi di laboratorio; iii) alimentare il sistema informativo per i programmi di controllo in sicurezza alimentare (SIPSA) tramite VETINFO; e iv) inviare al laboratorio nazionale di riferimento (c/o IZS Abruzzo e Molise) i ceppi isolati di L. monocytogenes.

Le categorie di prodotti da campionare erano rappresentati da formaggi a pasta molle o semi-molle $(n=40)$, prodotti a base di carne sottoposti a trattamento termico $(n=40)$ e pesce affumicato $(n=80)$.

Tutti i campioni pervenuti sono stati sottoposti contestualmente ad analisi qualitativa (secondo metodica ISO 11290-1; ISO, 1996) e quantitativa (secondo metodica ISO 11290-2; ISO, 1998) per la ricerca e il conteggio di $L$. monocytogenes. Le analisi sono state effettuate alla data di scadenza riportata in etichetta, ad esclusione della categoria pesce affumicato; per questa matrice, infatti, il piano prevedeva di effettuare un doppio campione per ciascuna delle 40 partite in modo da poter analizzare il primo campione entro $24 \mathrm{~h}$ dal suo arrivo in laboratorio e il secondo alla data di scadenza, rendendo così disponibili due risultati analitici utili per valutare il comportamento di $L$. monocytogenes durante la vita commerciale dei prodotti.

Inoltre, sempre sui campioni di pesce affumicato, poiché la crescita di L. monocytogenes è influenzata anche dal $\mathrm{pH}$ e dall' $\mathrm{a}_{\mathrm{w}}$, il programma di monitoraggio includeva anche la misurazione di tali parametri sul campione analizzato entro $24 \mathrm{~h}$ dall'arrivo in laboratorio.

\section{Risultati}

Le numerosità campionarie previste dal programma sono state rispettate per tutte le categorie incluse nel piano (le frequenze sono riportate nella Tabella 1). Le temperature di trasporto registrate all'arrivo del campione in laboratorio sono rientrate nell'intervallo richiesto, ovvero tra 2 e $8^{\circ} \mathrm{C}$. Per quanto riguarda i campioni in attesa di essere esaminati al termine del periodo di shelf-life, la temperatura di conservazione è stata compresa tra

\section{2 e $4^{\circ} \mathrm{C}$.}

Tutti i prodotti a base di carne e tutti i campioni di formaggio molle e semi-molle sono risultati conformi sia al conteggio, sia all'analisi qualitativa.

Per quanto riguarda il salmone affumicato (analizzate 40 partite costituite da 80 campioni): 7 campioni su $80(8,75 \%)$ appartenenti a 5 partite sono risultati positivi all'analisi qualitativa (presenza in $25 \mathrm{~g}$ ) mentre tutti i campioni $(80 / 80)$ sono risultati conformi all'analisi quantitativa (conteggi $<10 \mathrm{ufc/g}$ a fronte del limite di $100 \mathrm{ufc/g}$ fissato dal Reg. CE 2073/05 e ss.mm.ii.; Commissione Europea, 2005).

I risultati non conformi sono stati ottenuti, quindi, solo con la metodica qualitativa. In particolare: 4 campioni di 2 partite analizzati all'arrivo in laboratorio e alla fine della shelf life; 2 campioni di 2 partite analizzati alla fine della shelf-life; e 1 campione di 1 partita analizzato all'arrivo in laboratorio.

Per quanto attiene la misurazione del $\mathrm{pH}, \mathrm{si}$ sono registrati valori compresi tra 5.7 e 6.3 , mentre l' $a_{w}$ è risultata compresa tra 0,93 e 0,98 .

\section{Discussione}

I dati ottenuti dalle analisi effettuate sui formaggi e sui prodotti a base di carne sono confortanti, non avendo mai consentito di isolare il patogeno.

Per quanto riguarda il salmone affumicato, è importante ricordare come questo sia indicato tra gli alimenti maggiormente contaminati da L. monocytogenes (EFSA, 2009); è inoltre un prodotto ittico pronto al consumo che può essere quindi consumato tal quale e che può entrare come ingrediente in numerose preparazioni alimentari, quali sandwich, tramezzini e simili. Come alimento RTE, per le sue caratteristiche di $\mathrm{pH} \mathrm{e} \mathrm{a}_{\mathrm{w}}$, costituisce, secondo quanto indicato dal Reg. CE 2073/05 (Commissione Europea, 2005) e ss.mm.ii., terreno favorevole alla crescita di $L$. monocytogenes, rappresentando un rischio potenziale per i consumatori e in particolare per alcune categorie a rischio (bambini, anziani, donne in gravidanza, soggetti immunocompromessi); non a caso, il Reg. CE 2073/2005 (Commissione Europea, 2005) e ss.mm.ii. include tale batterio nei criteri di sicurezza per gli alimenti RTE, imponendo all'operatore del settore alimentare (OSA) controlli per la ricerca del patogeno, in grado di garantire che il livello di contaminazione non superi le $100 \mathrm{ufc} / \mathrm{g}$ al termine della sua vita commerciale.

I risultati ottenuti con la metodica qualitativa $(8,75 \%$ di campioni di salmone affumicato positivi) prospettano uno scenario poco confortante per quanto riguarda la contaminazione di tale matrice da parte di $L$. monocytogenes. Tuttavia, i risultati ottenuti con il metodo quantitativo, con conteggi sempre inferiori a $10 \mathrm{ufc} / \mathrm{g}$, ridimensionano il problema e l'effettivo rischio per il consumatore. Punto fondamentale per una valutazione delle informazioni ottenute è sicuramente il corretto mantenimento della catena del freddo; infatti, i campioni analizzati a fine vita commerciale, sono stati mantenuti a una temperatura di refrigerazione controllata. È importante quindi ricordare come ogni eventuale abuso termico possa portare a un aumento della carica batterica eventualmente presente.

\section{Conclusioni}

La scelta dell'analisi appropriata da eseguire nell'ambito dei controlli ufficiali per definire la conformità del prodotto ai sensi del

Tabella 1. Frequenze di prelievo per tipo di campione.

\begin{tabular}{llc} 
Categoria & Tipo campione & $\begin{array}{c}\text { Campioni previsti ed effettuati } \\
\text { (n. totale) }\end{array}$ \\
Pesce affumicato & Salmone affumicato & 80 \\
Formaggi molli e semi-molli & Brie & 3 \\
& Caciotta & 2 \\
& Camembert & 1 \\
& Gorgonzola & 12 \\
& Italico & 2 \\
& Quartirolo & 1 \\
& Stracchini e crescenze & 15 \\
& Taleggio & 4 \\
\hline Prodotti a base di carne sottoposti & Arrosto & 1 \\
a trattamento termico & Mortadella & 9 \\
& Prosciutto cotto & 29 \\
Totale & Spalla cotta & 1 \\
\hline
\end{tabular}


Reg. CE 2073/05 (Commissione Europea, 2005) e ss.mm.ii. presuppone la preliminare disponibilità di un'informazione fondamentale: le garanzie che il produttore è in grado di fornire dimostrando con soddisfazione dell'autorità competente che il proprio prodotto non supererà $100 \mathrm{ufc} / \mathrm{g}$ durante il periodo di conservabilità. Tali informazioni, utili non solo per l'autorità competente ma anche per l'OSA, dovrebbero essere ottenute dal produttore mediante specifici studi di shelf-life e challenge test.

La tipologia di analisi da eseguirsi e l'interpretazione dei risultati delle stesse dovrebbero essere sempre effettuati in relazione ai dati forniti dal produttore. Purtroppo, però, tali informazioni non sempre sono disponibili nella fase del prelievo del campione.

\section{Bibliografia}

Buchanan R, Lindqvist R, Ross T, Smith M, Todd E, Whiting R, 2004. Risk assessment of Listeria monocytogenes in ready-to-eat foods. Food and Agriculture Organization Pubbl., Roma, Italia.

Commissione Europea, 2005. Regolamento (CE) 2073/2005 della Commissione del 15 novembre 2005 sui criteri microbiologici applicabili ai prodotti alimentari,
2073/2005/CE. In: Gazzetta Ufficiale, L $338 / 1,22 / 12 / 2005$.

Commissione Europea, 2010. Gazzetta ufficiale dell'Unione europea: decisione della commissione del 5 novembre 2010 concernente una partecipazione finanziaria dell'Unione a un programma coordinato di sorveglianza sulla prevalenza di Listeria monocytogenes in taluni alimenti pronti, da realizzare negli Stati Membri, 2010/75/UE. In: Gazzetta Ufficiale, L 37/55, $10 / 2 / 2010$.

EFSA, 2009. Report of task force on zoonoses data collection 2009. Proposed technical specifications for a survey on Listeria monocytogenes in selected categories of ready to eat food at retail in the EU. EFSA Journal 300:1-66.

Gandhi M, Chikindas ML, 2007. Listeria: a foodborne pathogen that knows how to survive. Int J Food Microbiol 113:1-15.

Garrido V, Garcìa-Jalòn I, Vitas AI, 2010. Food Control 21:896-901.

ISO, 1996. Microbiology of food and animal feeding stuffs. Horizontal method for the detection and enumeration of Listeria monocytogenes. Part 1: detection method. Norma ISO 11290-1:1996. Organizzazione internazionale per la normazione ed., Ginevra, Svizzera.

IS0, 1998. Microbiology of food and animal feeding stuffs. Horizontal method for the detection and enumeration of Listeria monocytogenes. Part 2: enumeration method. Norma ISO 11290-2:1998. Organizzazione internazionale per la normazione ed., Ginevra, Svizzera.

Kalmokoff ML, Austin JWX, Wan D, Sanders G, Banerjee S, Farber JM, 2001. Adsorption, attachment and biofilm formation among isolates of Listeria monocytogenes using model conditions. J Appl Microbiol 91:72534.

Murru N, Mormile A, Barile M, Pezone G, 2011. Ricerca di L. monocytogenes in alimenti e frigoriferi di case di cura. Rivista dell'AIVI 1:71-4.

Nuvolosi R, Pedonese F, D’Ascenzi C, Rindi S, 2006. La valutazione del rischio da Listeria monocytogenes in alimenti pronti al consumo. In: Annali della Facoltà di Medicina veterinaria, LIX/20, pp 29-45. Disponibile al sito: http://eprints.adm.unipi.it/391/

Porsby CH, Vogel BF, Mohr M, Gram L, 2008. Influence of processing steps in cold-smoked salmon production on survival and growth of persistent and presumed nonpersistent Listeria monocytogenes. Int $\mathrm{J}$ Food Microbiol 122:287-95.

Rørvik LM, 2000. Listeria monocytogenes in the smoked salmon industry. Int J Food Microbiol 62:183-90. 